\section{Vol. 32, Issue 1-2, February 2009}

\section{Editorial}

7 The Difficult Trials

Herrstedt, J. (Odense)

Original Articles

10 Surgery of Colorectal Carcinoma in Patients Aged over 80

Falch, C.; Kratt, T.; Beckert, S.; Kirschniak, A.; Zieker, D.; Königsrainer, I.; Löb, S.; Hartmann, J.T.; Königsrainer, A.; Brücher, B.L.D.M. (Tübingen)

18 Multicenter Phase II Study of Pegylated Liposomal Doxorubicin in Combination with Vinorelbine as First Line Treatment in Elderly Patients with Metastatic Breast Cancer

Mlineritsch, B. (Salzburg); Schabel-Moser, R. (Graz); Andel, J. (Steyr) Fridrik, M. (Linz); Moik, M.; Mayer, P.; Russ, G.; Rass, C.; Greil, R. (Salzburg) on behalf of the Arbeitsgemeinschaft medikamentöse Tumortherapie Study Group (AGMT)

25 Quality of Medical Care in Colorectal Cancer in Germany

Kube, R. (Magdeburg); Ptok, H. (Magdeburg, Cottbus); Wolff, S.; Lippert, H. (Magdeburg); Gastinger, I. (Magdeburg, Cottbus); the Study Group Colon/Rectum Carcinoma (Primary Tumor)

30 Chemotherapy-Induced Nausea and Vomiting in the Treatment of Gastrointestinal Tumors and Secondary Prophylaxis with Aprepitant

Abbrederis, K.; Lorenzen, S.; Röthling, N.; Ihbe-Heffinger, A.; Schuster, T.; Peschel, C. (München); Lordick, F. (Heidelberg)

35 Efficacy and Toxicity of ${ }^{153}$ Samarium-EDTMP in Painful Breast Cancer Bone Metastases Dolezal, J. (Hradec Kralove)

\section{Clinical Cases}

40 Reversible Foetal Cerebral Ventriculomegaly and Cardiomyopathy under Chemotherapy for Maternal AML

Baumgärtner, A.K.; Oberhoffer, R.; Jacobs, V.R.; Ostermayer, E.; Menzel, H.; Voigt, M.; Schneider, K.T.M.; Pildner von Steinburg, S. (München)

44 Sorafenib Induces Therapeutic Response in a Patient with Metastatic Collecting Duct Carcinoma of Kidney Ansari, J.; Fatima, A.; Chaudhri, S.; Bhatt, R.I.; Wallace, M.; James, N.D. (Birmingham)

\section{Band 32, Heft 1-2, Februar 2009}

Editorial

7 Die schwierigen Tests Herrstedt, J. (Odense)

Originalarbeiten

10 Chirurgische Therapie kolorektaler Karzinome bei Patienten über 80 Jahren

Falch, C.; Kratt, T.; Beckert, S.; Kirschniak, A.; Zieker, D.; Königsrainer, I.; Löb, S.; Hartmann, J.T.; Königsrainer, A.; Brücher, B.L.D.M. (Tübingen)

18 Multizentrische Phase-II-Studie mit pegyliertem liposomalen Doxorubicin in Kombination mit Vinorelbin als Erstlinientherapie des fortgeschrittenen Mammakarzinoms bei älteren Patientinnen Mlineritsch, B. (Salzburg); Schabel-Moser, R. (Graz); Andel, J. (Steyr); Fridrik, M. (Linz); Moik, M.; Mayer, P.; Russ, G.; Rass, C.; Greil, R. (Salzburg) on behalf of the Arbeitsgemeinschaft medikamentöse Tumortherapie Study Group (AGMT)

25 Qualität der medizinischen Versorgung des kolorektalen Karzinoms in Deutschland

Kube, R. (Magdeburg); Ptok, H. (Magdeburg, Cottbus); Wolff, S.; Lippert, H. (Magdeburg); Gastinger, I. (Magdeburg, Cottbus); the Study Group Colon/Rectum Carcinoma (Primary Tumor)

30 Chemotherapie-induzierte Übelkeit und Erbrechen in der Behandlung von Tumoren des Gastrointestinaltraktes und Sekundärprophylaxe mit Aprepitant

Abbrederis, K.; Lorenzen, S.; Röthling, N.; Ihbe-Heffinger, A.; Schuster, T.; Peschel, C. (München); Lordick, F. (Heidelberg)

35 Effizienz und Toxizität von ${ }^{153}$ Samarium-EDTMP bei schmerzhaften ossären Brustkrebsmetastasen Dolezal, J. (Hradec Kralove)

Kasuistiken

40 Reversible fetale Hirnventrikulomegalie und Kardiomyopathie unter Chemotherapie bei mütterlicher AML

Baumgärtner, A.K.; Oberhoffer, R.; Jacobs, V.R.; Ostermayer, E.; Menzel, H.; Voigt, M.; Schneider, K.T.M.; Pildner von Steinburg, S. (München)

44 Sorafenib induziert Therapieansprechen bei einem Patienten mit metastasiertem Sammelrohrkarzinom der Niere

Ansari, J.; Fatima, A.; Chaudhri, S.; Bhatt, R.I.; Wallace, M.; James, N.D. (Birmingham)

\section{KARGER}

Fax +497614520714

Information@Karger.de

www.karger.com 


\section{Vol. 32, Issue 1-2, February 2009}

47 Hürthle Cell Carcinoma of the Thyroid with Contralateral Malignant Pleural Effusion

Hsu, K.F.; Hsieh, C.B. (Taipei); Duh, Q.Y. (San Francisco, CA); Chien, C.F.; Li, H.S.; Shih, M.L. (Taipei)

50 Remission of Paraneoplastic Dermatomyositis Associated with Hepatocellular Carcinoma under Prednisolone and Azathiopin, and Concommittant Sorafenib

Apostolidis, L.; Kahlert, C.; Siegmund, A.; Thom, R.; Horstmann, S.; Jäger, D.; Lordick, F. (Heidelberg)

54 Peliosis Hepatis in Cancer Patients Mimicking Infection and Metastases

Wannesson, L.; Chigrinova, E.; Raditchkova, M. (Bellinzona); Mazzucchelli, L. (Locarno); Ghielmini M. (Bellinzona)

Review Article

57 Lymph Node Dissection in Rectal Carcinoma: TME and What Else?

Göhl, J.; Hohenberger, W.; Merkel, S. (Erlangen)

Reports of Oncological Societies

62 DGHO-Frühjahrstagung 2009 / Förderpreis

Geriatrische Onkologie 2008

Deutsche Gesellschaft für Hämatologie und Onkologie e. V. (DGHO)

63 PharmaForum

67 PharmaNews / PharmaTicker

71 Meetings and Conferences

74 Guidelines for Authors

34 Imprint

Forthcoming papers are listed on page 76.

\section{Band 32, Heft 1-2, Februar 2009}

47 Hürthle-Karzinom der Schilddrüse mit kontralateralem malignem Pleuraerguss

Hsu, K.F.; Hsieh, C.B. (Taipei); Duh, Q.Y. (San Francisco, CA); Chien, C.F.; Li, H.S.; Shih, M.L. (Taipei)

50 Remission von paraneoplastischer Dermatomyositis assoziiert mit hepatozellulärem Karzinom während der Behandlung mit Prednisolon und Azathioprin und gleichzeitiger Sorafenib-Therapie Apostolidis, L.; Kahlert, C.; Siegmund, A.; Thom, R.; Horstmann, S.; Jäger, D.; Lordick, F. (Heidelberg)

54 Peliosis hepatis bei Karzinom-Patienten - mögliche Fehldiagnose als Infektionen oder Metastasen Wannesson, L.; Chigrinova, E.; Raditchkova, M. (Bellinzona); Mazzucchelli, L. (Locarno); Ghielmini M. (Bellinzona)

Übersichtsarbeit

57 Lymphknotendissektion beim Rektumkarzinom: TME und was noch?

Göhl, J.; Hohenberger, W.; Merkel, S. (Erlangen)

Mitteilungen onkologischer Gesellschaften

62 DGHO-Frühjahrstagung 2009 / Förderpreis Geriatrische Onkologie 2008

Deutsche Gesellschaft für Hämatologie und Onkologie e. V. (DGHO)
63 PharmaForum

67 PharmaNews / PharmaTicker

71 Tagungen und Kongresse

75 Hinweise für Autoren

34 Impressum
Einen Ausblick auf den Inhalt der kommenden Hefte finden Sie auf Seite 76.

\section{KARGER}

Fax +497614520714

Information@Karger.de

www.karger.com (c) 2009 S. Karger GmbH, Freiburg 